\title{
Shell model description of heavy nuclei and abnormal collective motions
}

\author{
Chong $\mathrm{Qi}^{1, *}$ \\ ${ }^{1}$ KTH Royal Institute of Technology, SE-10691 Stockholm
}

\begin{abstract}
In this contribution I present systematic calculations on the spectroscopy and electromagnetic transition properties of intermediate-mass and heavy nuclei around ${ }^{100} \mathrm{Sn}$ and ${ }^{208} \mathrm{~Pb}$. We employed the large-scale configuration interaction shell model approach with realistic interactions. Those nuclei are the longest isotopic chains that can be studied by the nuclear shell model. I will show that the yrast spectra of Te isotopes show a vibrational-like equally spaced pattern but the few known E2 transitions show rotational-like behaviour. These kinds of abnormal collective behaviors cannot be reproduced by standard collective models and provide excellent background to study the competition of single-particle and various collective degrees of freedom. Moreover, the calculated B(E2) values for neutron-deficient and heavier Te isotopes show contrasting different behaviours along the yrast line, which may be related to the enhanced neutron-proton correlation when approaching $\mathrm{N}=50$. The deviations between theory and experiment concerning the energies and $\mathrm{E} 2$ transition properties of low-lying $0^{+}$and $2^{+}$excited states and isomeric states in those nuclei may provide a constraint on our understanding of nuclear deformation and intruder configuration in that region.
\end{abstract}

From a nuclear structure physics point of view, one also aims at addressing fundamental nuclear physics questions including: How are complex nuclei built from their basic constituents and how to explain collective phenomena from individual motion [1]? Nuclear theory plays a critical role in explaining those regular emerging phenomena in nuclear many-body systems. In particular, the nuclear shell-model calculations have been shown to be very successful in describing nuclei below ${ }^{100} \mathrm{Sn}$. The aim of our work is to provide a microscopic shell-model description of the structure and collective behaviours of intermediate-mass and heavy nuclei. In particular, I will show its application of the shell model in nuclei around ${ }^{100} \mathrm{Sn}$ and ${ }^{208} \mathrm{~Pb}$. I will explain the structure and decay studies of those nuclei, regarding both experimental and theoretical opportunities. Although not covered in this contribution, it may be useful to mention that a proper description of the neutron-rich isotones around $N=82$ and 126 can be important for our understanding of the astrophysical rapid neutron capture process and its possible existence of the third peak.

Nuclear structure models treat the mean field in quite different ways. Within the ab initio family, the no-core shell model approach aims at considering the residual correlation between all nuclei in a large space defined by the harmonic oscillator single-particle orbitals. The nuclear shell model, as we call it, is a full configuration interaction approach. It considers the mixing effect of all possible configurations within a given model space. The model space is usually defined by taking a few single-particle orbitals near the Fermi surface. The number of orbitals one can include in above two models is highly restricted

*e-mail: chongq@kth.se due to computation limitation. Extensive studies on algorithm optimization and possible truncation or approximation methods have been carried out. State-of-the-art configuration interaction algorithms are able to diagonalize matrices with dimension up to $2 \times 10^{10}$. Other challenges of the no-core and full configuration interaction shell model approaches include for examples the connection of their effective interaction to fundamental forces and the complexity of the their wave function. Despite of these challenges, the nuclear shell model is by far the most accurate and precise theory available on the market.

The residual interaction between valence particles around the Fermi surface is mostly supposed to be of twobody nature. The two-body matrix elements of the effective Hamiltonian can be calculated from realistic nucleonnucleon potential where one has to consider the effect of its short-range repulsion and the core polarization effects induced by the assumed inert core. However, an optimization of the monopole interaction is necessary in most cases due to the neglect of explicit three-body force and other effects. We have optimized the monopole channel of the effective Hamiltonians for describing the $\mathrm{Sn}$ and $\mathrm{Pb}$ isotopes, which work well also for most open-shell nuclei. We consider single-particle orbitals $g_{07 / 2}, 1 d_{5 / 2,3 / 2}, 2 s_{1 / 2}$ and $0 h_{11 / 2}$ (simply denoted as gdsh) for our studies of nuclei above ${ }^{100} \mathrm{Sn}[2]$ and the six orbitals between $N=82$ and $126,0 h_{9 / 2}, 1 f_{7 / 2,5 / 2}, 2 p_{3 / 2,1 / 2}$ and $0 i_{13 / 2}$ (denoted as hfpi) for our studies of neutron-deficient $\mathrm{Pb}$ isotopes [3]. But it should be pointed out that the motivation is not to fit data better but to get a better wave function and more reliable predictions on unknowns. We also applied those two effective interactions together for our studies of nuclei involving protons in gdsh and neutrons in hfpi shells. 
The neutron single-particle states $d_{5 / 2}$ and $g_{7 / 2}$ orbitals in ${ }^{101} \mathrm{Sn}$ are expected to be very close to each other. Our Hamiltonian works well also for $\mathrm{Sb}, \mathrm{I}$ and $\mathrm{Xe}$ isotopes and heavier isotopes $[4,5]$ isotopes. As examples, in Fig. 1 I plotted the partial calculated level schemes of ${ }^{112,132} \mathrm{Ba}$ (with 6 protons being coupled to 6 neutrons/neutron holes) which are the largest systems we have ever treated in the full/non-truncated shell model space calculation. As can be seen from the figure, the calculations reproduce rather well the experimental spectrum of ${ }^{132} \mathrm{Ba}$. It is interesting to see that the calculated spectra of ${ }^{112,132} \mathrm{Ba}$ are quite close to each other, even though the former case is expected to be highly influenced by the strong neutron-proton interaction. We are also optimizing the $T=0$ channel of the effective interaction. In addition, we are extending the model space to include orbitals between the $N=Z=50$ shell closure as well as neutron orbitals above $N=82$ in order to study the structure of neutron-rich Cd isotopes.

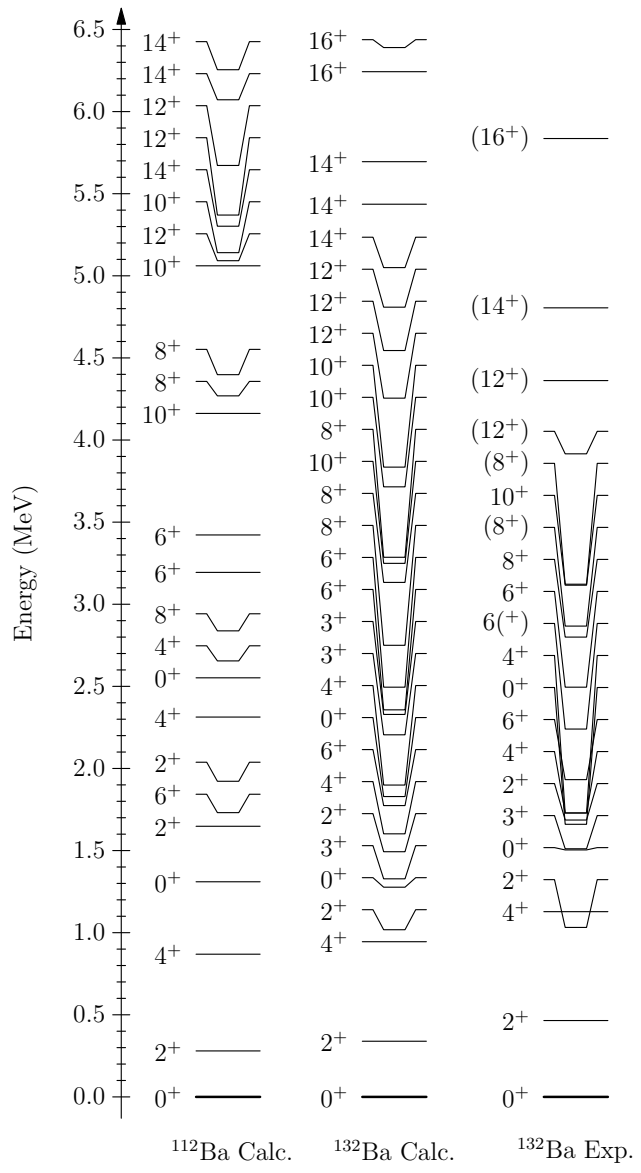

Figure 1. Calculated partial level schemes for ${ }^{112} \mathrm{Ba}(6 \pi-6 v)$ and ${ }^{132} \mathrm{Ba}(6 \mathrm{p}-6 \mathrm{~h})$ in comparison with experimental data on ${ }^{132} \mathrm{Ba}$. We calculated the lowest few states for selected spin values and positive parity.
It was argued that ${ }^{100} \mathrm{Sn}$ may be a soft core in analogy to the soft $N=Z=28$ core ${ }^{56} \mathrm{Ni}$. It seems such a possibility can be ruled out based on indirect information from recent measurements in this region [6-10]. The asymmetric electric quadrupole (E2) transition shape in $\mathrm{Sn}$ isotopes is suggested to be induced by the Pauli blocking effect [6]. A systematic study on the E2 transition in Te isotopes is done in Ref. [10]. One can state that the limited number of valence protons and neutrons are not expected to induce any significant quadrupole correlation in this region [1114].

Truncations often have to be applied in order to reduce the size of the shell-model bases. The simplest way of truncation is to restrict the maximal/minimal numbers of particles in different orbitals. This method is applied both to no-core (often being referred to Nmax) and empirical shell model (npnh) calculations. In Ref. [6] we studied the structure and electromagnetic transition properties of light $\mathrm{Sn}$ isotopes within the large $g d s h_{11 / 2}$ model space by restricting the maximal number of four neutrons that can be excited out of the $g_{9 / 2}$ orbital. However, the convergence can be very slow if there is no clear shell or subshell closure or if single-particle structure are significantly modified by the monopole interaction, as it happens in neutronrich light nuclei (see, e.g., Ref. [15]). One can evaluate the importance of a given basis vector $\psi_{i}$ within a partition through a perturbation measure. The challenge here is that the truncated bases may not conserve angular momentum. An angular momentum conserved correlated basis truncation approach is introduced in Ref. [16]. We are implementing this method in the widely distributed shell-model code NuShellX by replacing its projection subroutine with our new correlated basis method. We have also developed an importance truncation based on the total monopole energy. The idea behind is that the Hamiltonian is dominated by the diagonal monopole channel. The monopole interaction can change significantly the (effective) mean field and drive the evolution of the shell structure. We have done truncation calculations for two isotopes ${ }^{200,194} \mathrm{~Pb}$. Convergence can be reached with a small portion (around 10\%) of the total M-scheme wave function in both cases. This truncation approach can be easily implemented in most existing shell model codes [17].

We have done a systematic calculation on the structure and electromagnetic transition properties of neutrondeficient $\mathrm{Pb}$ isotopes, which may be studied experimentally using the AGATA detector. Our shell-model calculations can reproduce well the excitation energies of the low-lying $0^{+}$and $2^{+}$states in isotopes ${ }^{198-206} \mathrm{~Pb}$ [3]. The overall agreement between experiments and calculations are quite satisfactory. For nuclei heavier than ${ }^{196} \mathrm{~Pb}$, the difference between theory and experiment is less than 100 $\mathrm{keV}$. In addition, one can also reproduce well the binding energies as well as the empirical pairing gaps. The empirical pairing gaps can be extracted from the binding energy by using the simple three-point formula, which carry important information on the two-nucleon pair clustering as well as $\alpha$ clustering in the nuclei involved [18, 19]. In nuclear systems the pairing collectivity manifests itself through the coherent contribution of many shell-model 
configurations which is induced by the strong $T=1$ pairing interaction. For $\mathrm{Pb}$ isotopes, the full shell-model results also agree well with our generalized seniority and nucleon-pair-approximation truncation calculations.

The deviations between theory and experiment concerning the excitation energies and electromagnetic properties of low-lying $0^{+}$and $2^{+}$excited states in $\mathrm{Pb}$ isotopes indicate that, as suggested by deformed mean field calculations, nuclear deformation as induced intruder configurations from outside the model space should be important for nuclei in that region. Large-scale calculations are being carried out by including by neutron and proton core excited configuration. The excitation energies of the first $2^{+}$isotopes in $\mathrm{Pb}$ isotopes show a rather weak parabolic behaviour. In the lighter $\mathrm{Pb}$ isotopes the excitation energy of the second $0^{+}$state decreases rapidly with decreasing neutron number. It even becomes the first excited state in ${ }^{184-194} \mathrm{~Pb}$. Within a shell-model context, those low-lying $0^{+}$states may be interpreted as coexisting deformed states which are induced by proton pair excitations across the $Z=82$ shell gap. The energy of those core-excited configurations get more favoured in mid-shell $\mathrm{Pb}$ isotopes in relation to the stronger neutron-proton correlation in those nuclei (see talks by Garcia-Ramos and Andreyev). The shape-coexisting structure of $\mathrm{Cd}$ and $\mathrm{Te}$ isotopes is described in Garrett's talk.

In addition to seniority, it is well known that atomic nuclei can exhibit regular and strongly collective motions like vibration and rotation. The rotation and the underlying nuclear deformation in intrinsic frame has been well described by the nuclear shell model. It has its root in the quadrupole-quadrupole neutron-proton correlation. On the other hand, much less is known on the microscopic orgin of the vibration. The low-lying collective excitations of mid-shell Cd and Te isotopes were discussed in terms of quadrupole vibrations $[11,20]$ in relation to the fact that the even-even isotopes between $N=56$ and 70 show regular equally-spaced yrast spectra. If that is the case, those isotopes will provide an ideal ground to explore the nature of the elusive nuclear vibration. Another intriguing phenomenon is the nearly constant behaviour of the energies of the $2^{+}$and $4^{+}$states in Te and Xe isotopes and their ratios when approaching $N=50$, in contrast to the decreasing behaviour when approaching $N=82$ [11]. This was analysed in Ref. [21] based on the quasiparticle random phase approximation approach.

Moreover, the available E2 transition strengths along the yrast line in ${ }^{114,120-124} \mathrm{Te}$ show an anomalous rotational-like behaviour [22, 23]. It cannot be reproduced by collective models or the interacting boson model. We have done systematic calculations on the E2 transition properties of Te isotopes in Ref. [24]. The calculations reproduce well the excitation energies of the lowlying states as well as the regular and vibrational-like behaviour of the yrast specta of ${ }^{108-130} \mathrm{Te}$. In particular, the calculations reproduced reasonably well the nearly constant behaviour of the $B(E 2)$ values of ${ }^{114} \mathrm{Te}$ and ${ }^{120-124} \mathrm{Te}$ along the yrast line. The anomalous constant behaviour is related to the competition between the seniority coupling and the neutron-proton correlations. On the other

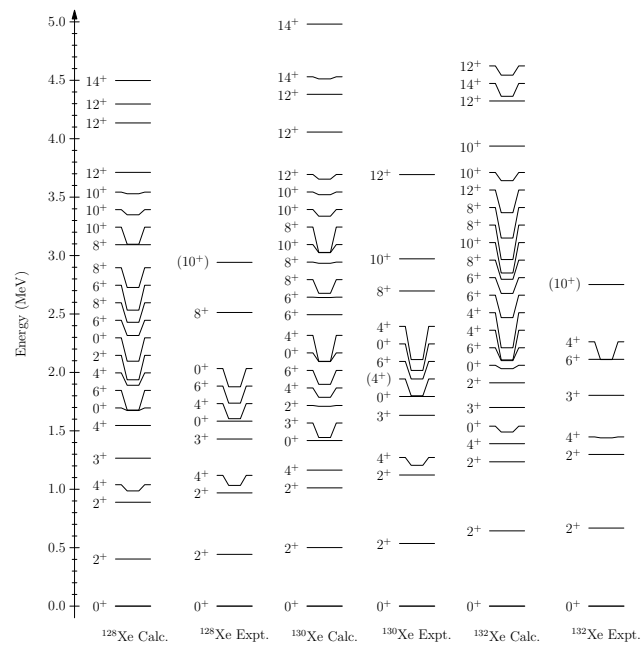

Figure 2. Calculated partial level schemes of ${ }^{128-132} \mathrm{Xe}$ in comparison with expeirmetal data.

hand, a squeezed gap between the $6_{1}^{+}$and $4_{1}^{+}$states is seen in Te isotopes when approaching $N=82$, resulting in seniority-like spectra. A further experiment is done to study the angular momentum dependence of the E2 transition strengths in ${ }^{112} \mathrm{Te}$ [25]. It shows a similar but more 'vibrational'-like behaviour. Potential-Energy Surface calculations [13] indicate that those nuclei may also be understood from a soft rotor point of view. The yrast spectra of Te isotopes show a vibrational-like equally spaced pattern but the few known E2 transitions show anomalous rotational-like behaviour, which cannot be reproduced by collective models. Moreover, the calculated B(E2) values for neutron-deficient and heavier Te isotopes show contrasting different behaviours along the yrast line, which may be related to the enhanced neutron-proton correlation when approaching $N=50$.

There have been extensive studies on the possible existence of $\mathrm{E}(5)$ symmetry in nuclei around $A=130$. The nucleus ${ }^{134} \mathrm{Ba}$ may be the best $\mathrm{E}(5)$ candidate whereas the possibility to see that symmetry in $\mathrm{Xe}$ isotopes may have been ruled out in relation to the unexpected observation on the E2 transition properties between nonyrast $2^{+}$and $0^{+}$states. We are analysing the calculations on the E2 transition properties of those nuclei. In Fig. 2 we plotted the calculated low-lying states of ${ }^{128-132} \mathrm{Xe}$ and compared with experimental data.

Further calculations on the E2 transitions of Cd isotopes (with two proton holes instead of particles within $Z=50$ closure) are underway. The structure properties of Cd isotopes were also reviewed in also Garrett's and Leviatan's talks. The lighter deformed Cd isotopes was studied recently within the shell-model framework in Ref. [26].

To summarize, we have presented selected shell-model results on semi-magic $\mathrm{Sn}$ and $\mathrm{Pb}$ isotopes and open-shell 
nuclei including $\mathrm{Te}$ and $\mathrm{Ba}$ isotopes. I hope that the shell model can be a reliable tool for studying the spectroscopy and (abnormal) collective behaviours of those intermediate mass and heavy nuclei. In the near future, we are in particular interested to have a full understanding the vibrational behaviour of the $\mathrm{Cd}$ isotopes as well as the critical-point symmetry behaviours in $A \sim 130$ region.

This work is supported by the Swedish Research Council (VR) under grant Nos. 621-2012-3805, and 6212013-4323 and the Göran Gustafsson foundation. Computational support provided by the Swedish National Infrastructure for Computing (SNIC) at PDC, KTH, Stockholm is acknowledged. The author also acknowledge the support from KTH through its strategic partnership with Shanghai JiaoTong University.

\section{References}

[1] Nupecc long range plan 2017 "perspectives for nuclear physics", http://www.nupecc.org/ lrp2016/Documents/lrp2017.pdf

[2] C. Qi, Z.X. Xu, Phys. Rev. C 86, 044323 (2012)

[3] C. Qi, L.Y. Jia, G.J. Fu, Phys. Rev. C 94, 014312 (2016)

[4] F. Wang, B.H. Sun, Z. Liu, C. Qi, L.H. Zhu, C. Scholey, S.F. Ashley, L. Bianco, I.J. Cullen, I.G. Darby et al., Phys. Rev. C 96, 064307 (2017)

[5] F. Wang, B. Sun, Z. Liu, R. Page, C. Qi, C. Scholey, S. Ashley, L. Bianco, I. Cullen, I. Darby et al., Phys. Lett. B 770, 83 (2017)

[6] T. Back, C. Qi, B. Cederwall, R. Liotta, F.G. Moradi, A. Johnson, R. Wyss, R. Wadsworth, Phys. Rev. C 87, 031306 (2013)

[7] T. Bäck et al., Phys. Rev. C 84, 041306 (2011)
[8] C.B. Hinke et al., Nature 486, 341 (2012)

[9] G. Guastalla et al., Phys. Rev. Lett. 110, 172501 (2013)

[10] M. Doncel et al., Phys. Rev. C 91, 061304 (2015)

[11] B. Hadinia et al., Phys. Rev. C 72 (2005)

[12] E. Caurier, F. Nowacki, A. Poves, K. Sieja, Phys. Rev. C 82, 064304 (2010)

[13] Z.Y. Wu, C. Qi, R. Wyss, H.L. Liu, Phys. Rev. C 92, 024306 (2015)

[14] E.A. Coello Pérez, T. Papenbrock, Phys. Rev. C 92, 064309 (2015)

[15] Z.X. Xu, C. Qi, Phys. Lett. B 724, 247 (2013)

[16] L.F. Jiao, Z.H. Sun, Z.X. Xu, F.R. Xu, C. Qi, Phys. Rev. C 90, 024306 (2014)

[17] C. Qi, Journal of Physics: Conference Series 742, 012030 (2016)

[18] A.N. Andreyev et al., Phys. Rev. Lett. 110, 242502 (2013)

[19] C. Qi, A. Andreyev, M. Huyse, R. Liotta, P.V. Duppen, R. Wyss, Phys. Lett. B 734, 203 (2014)

[20] Z. Dombrádi et al., Phys. Rev. C 51, 2394 (1995)

[21] D.S. Delion, R. Wyss, R.J. Liotta, B. Cederwall, A. Johnson, M. Sandzelius, Phys. Rev. C 82, 024307 (2010)

[22] O. Möller, N. Warr, J. Jolie, A. Dewald, A. Fitzler, A. Linnemann, K.O. Zell, P.E. Garrett, S.W. Yates, Phys. Rev. C 71, 064324 (2005)

[23] M. Saxena et al., Phys. Rev. C 90, 024316 (2014)

[24] C. Qi, Phys. Rev. C 94, 034310 (2016)

[25] M. Doncel, T. Bäck, C. Qi, D.M. Cullen, D. Hodge, B. Cederwall, M.J. Taylor, M. Procter, M. Giles, K. Auranen et al., Phys. Rev. C 96, 051304 (2017)

[26] T. Schmidt, K.L.G. Heyde, A. Blazhev, J. Jolie, Phys. Rev. C 96, 014302 (2017) 